\title{
Prediction of Ovarian Response with Ovarian Response Prediction Index (Orpi) during Controlled Ovarian Stimulation in IVF
}

\author{
Mannem Haritha. M', Agarwal Sonal. A², Nayak Chaitra ${ }^{3}$, Pragnesh Gautham ${ }^{4}$, Kamini A \\ Rao. 5
}

${ }^{1}$ MBBS, MS (OBG), FMAS, FRM (REP MED) Infertility Specialist, Department of reproductive medicine, Milann Fertility Centre, Bangalore Assisted Conception Centre, Bengaluru, India

${ }^{2}$ MBBS,MS (OBG),FMAS,MNAMS,DNB,FNB(RM) Infertility Specialist, Department of reproductive Medicine, Milann Fertility Centre, Bangalore Assisted Conception Centre, Bengaluru, India

${ }^{3}$ MBBS, MS (OBG), FNB (RM) Infertility Specialist, Department of Reproductive Medicine, Milann Fertility Centre, Bangalore Assisted Conception Centre, Bengaluru, India

${ }^{4}$ MBBS, MD, DNB, Chief Operating Officer, Department of Reproductive Medicine, Milann Fertility Centre, Bangalore Assisted conception Centre, Bengaluru, India

${ }^{5}$ DGO, Dch, FICOG, FRCOG, PGDMLE, FNAMS, Medical Director, Department of Reproductive Medicine, Milann Fertility Centre, Bangalore Assisted Conception Centre, Bengaluru, India

Published: 20/09/2020

\begin{abstract}
(Background): To evaluate ORPI as an index to predict the response to ovarian stimulation. (Methods): It is an observational prospective study of 734 patients who underwent controlled ovarian stimulation during period of 1.5 years (July 2017 to December 2018) .Inclusion and exclusion criteria were taken into consideration when patients were recruited. ORPI is calculated by multiplying AMH level (ng/ml) and AFC (n) and the result is divided by age (years) of the patient. The primary outcome measured was number of MII oocytes and secondary outcome was total number of oocytes retrieved. (Results): Positive correlation of ORPI with MII oocytes and total number of oocytes is seen. Regarding the probability of collecting $\geq 4$ oocytes under the ROC curve, the AUC for ORPI is 0.68 (95\%CI 0.65-0.72) with sensitivity of 78.4 and specificity of 51.4 for a cut off of $>0.44$. For collecting $\geq 15$ oocytes ROC curve had an AUC of 0.72 with sensitivity of 66.7 and specificity of 73.4 for a cut off of $>1.28$. ROC curve for the probability of collecting $\geq 4$ MII oocytes depicted an AUC of 0.67 with cut off of $>0.77$. (Conclusion): The results of our study concluded that in a patient undergoing IVF treatment, ORPI has a poor ability to predict retrieval of $\geq 4$ oocytes or $\geq 4$ MII and fair ability for hyper response with $\geq 15$ oocytes. ORPI can serve as a counselling tool for predicting ovarian response.
\end{abstract}

Keywords: Antral follicle count, Anti-Mullerianhormone, Controlled ovarian stimulation

\section{Introduction}

Different protocols are present for multi follicular development in IVF so as to increase the number of embryos available and decrease time to pregnancy. Hypo response or hyper response cannot be predicted always. Supra physiological oestrogen levels result from large number of follicles which in turn has a negative effect on embryo quality and endometrium (1). Clinicians should individualise the gonadotropin dosage to reduce adverse effects of excessive ovarian response or poor response. Various predictors exist to predict ovarian response such as age, anti-mullerian hormone (AMH), antral follicle count (AFC), ovarian volume, day 2 follicle stimulating hormone, estradiol, inhibin and dynamic tests. Out of these age, AMH and AFC have served in the most effective way (2).

Oocyte number and quality decreases with age with dissimilarities in different races resulting in different responses to ovarian stimulation (3).Chronological age cannot

*Corresponding author: Haritha Mannem, ${ }^{1} \mathrm{MBBS}$, MS (OBG), FMAS, FRM (REP MED) Infertility Specialist, Department of reproductive medicine, Milann Fertility Centre, Bangalore Assisted Conception Centre, Bengaluru, India. Email: : harithamannem88@gmail.com serve as predictor of ovarian response so biological age as predicted by hormonal and functional profiles should be taken into consideration (4). Antral follicle count which is measure of follicles of $2-9 \mathrm{~mm}$ in both ovaries on day $2 / 3$ of menstrual cycle seen on trans-vaginal ultrasound is also being used as a predictor of ovarian response (5). Limitation to it is that there is subjective variation with cycle to cycle variability. $\mathrm{AMH}$, a member of the transforming growth factor-beta superfamily, is secreted by granulosa cells of pre antral and small antral follicles (6). Anti-mullerian hormone is a direct indicator of ovarian reserve and is independent of follicle stimulating hormone (FSH). AMH has no cycle variability and decreases throughout reproductive life to become undetectable in postmenopausal period. Thus in a nutshell all markers have errors in their estimation. Systematic reviews of ovarian reserve tests have depicted modest accuracy of all ovarian reserve tests for prediction of both hyper ovarian response and poor ovarian response when calculated individually (7).

Considering advantages like ability to calculate starting dose of gonadotropins, decreasing iatrogenic complications and cancellation rate and improving cost benefit ratio of ovarian stimulation protocols, our study has used a new ovarian response prediction index (ORPI) to assess the response to ovarian stimulation (8). 
ORPI ((ovarian response prediction index) is based on three ovarian reserve markers i.e. AMH, AFC and age to serve as a predictor for ovarian response during controlled ovarian stimulation in IVF. ORPI [AMH (ng/ml) x AFC (2-9 $\mathrm{mm}) /$ patient age] is being entitled to predict optimal ovarian response of $\geq 4$ oocytes and hyper response i.e. $\geq 15$ oocytes efficiently (9). ORPI has a cost benefit ratio in favour of benefit as it guides in individualising treatment and serves as a counselling tool for the couple regarding their predicted ovarian response.

\section{Materials and Methods \\ 2.1 Population}

In the current study inclusion criteria were: age $\leq 35$ years, body mass index (BMI) between $20-30 \mathrm{~kg} / \mathrm{m} 2$, regular menstrual cycles and both ovaries present. Exclusion criteria were: History of ovarian surgery, severe endometriosis, endocrine disorders, and presence of ovarian cysts assessed by trans-vaginal ultrasound.

Total 734 patients undergoing autologous IVF cycles were recruited for the study in the duration of 18 months. The study was reviewed by local ethical committee and clearance obtained from Institutional Review Board. Written informed consent was taken from all recruited patients. ORPI was calculated by multiplying AMH level $(\mathrm{ng} / \mathrm{ml})$ and AFC (n) and dividing it by age (years) of the patient.

\subsection{AMH measurement}

Venous blood was collected irrespective of day of menstrual cycle and AMH was measured using an enzymatically amplified 2-site immunoassay kit (AMH Gen II ELISA, Beckman Coulter Inc.). The lowest detection limit of this assay is $0.01 \mathrm{ng} / \mathrm{ml}$, whereas the maximum intra- and inter-assay coefficients of variation are $3.3 \%$ and $6.5 \%$, respectively.

\section{3 antral follicle count}

Transvaginal ultrasound (5.5-7 MHz) was done on day 2/3 of menstrual cycle by clinician who was blinded to the AMH value and other hormonal parameters. Follicles of 2-9 mm size were measured in both ovaries and total count was labelled as antral follicle count.

\subsection{Controlled ovarian stimulation}

Prior to starting ovarian stimulation, baseline scan by trans-vaginal ultrasound (Voluson P6) using vaginal probe was performed and follicle stimulating hormone (FSH), luteinizing hormone $(\mathrm{LH})$, estradiol (E2), progesterone (P4), anti-mullerian hormone (AMH) were done on day 2.Controlled ovarian stimulation (COS) was started on day $2 / 3$ of cycle with either recombinant Follicle stimulating hormone (R-FSH), (Recagon, Organon; Gonal F, Merck) with or without human menopausal gonadotropin (hMG; Menopur; Ferring Pharmaceuticals, Parsippany, NJ). Starting dose was calculated based on age, BMI, AFC, AMH and baseline FSH level. Ovarian response to stimulation was monitored during IVF cycle with trans-vaginal ultrasound and serum E2, LH and P4 measurements. Dose of gonadotropins was adjusted accordingly. Antagonist (Cetrotide, Merck) $0.25 \mathrm{mg}$ subcutaneously by flexible antagonist protocol was added when leading follicle was $\geq 13-14 \mathrm{~mm}$ in diameter or serum E2 > 350-400 pg/mL and was continued until trigger day. Patient was given hCG trigger injection when criteria of atleast 3 follicles $\geq 17 \mathrm{~mm}$ as mean diameter was attained.
Transvaginal oocyte retrieval was performed 34-36 hours post trigger under intravenous sedation. Number of oocytes retrieved and number of mature oocytes were noted.

\subsection{Endpoints}

The primary outcome measured was number of MII oocytes and secondary outcome was total number of oocytes retrieved. ORPI was calculated for retrieval of $\geq 4$ oocytes (adequate response), $\geq 15$ oocytes (hyper response) and number of MII oocytes.

\subsection{Calculation of Ovarian Response Prediction Index (ORPI)}

The ORPI value was calculated by multiplying the $\mathrm{AMH}$ (ng/ml) level by the AFC, and the result was divided by the age (years) of the patient: $\mathrm{ORPI}=(\mathrm{AMH} \times \mathrm{AFC}) /$ patient age . This definition of ORPI was based on Oliveira et al. (2012) study. The cut-off value was calculated by statistical analysis. This equation is based on previous evaluations that found that ovarian response to stimulation had positive correlations with AMH levels and number of antral follicles and was negatively correlated with patient's age (10). Notably, the calculated value of the ORPI in the study was not influenced by the protocol choice for the induction of ovulation or the doses of gonadotropin (11).

\subsection{Statistical analysis}

Analysis was done using SPSS software .Mann-Whitney test and chi-square test were used where appropriate. Correlations were performed using Pearson's correlation test. $\mathrm{P}<0.05$ was considered statistically significant. Univariate logistic regression module was used to estimate the value in predicting the likelihood of collecting $\geq 4$ oocytes, $\geq 4$ MII oocytes and $\geq 15$ oocytes. The odds ratio (OR) and 95\% confidence interval (CI) constituted the descriptive analysis. Receiver operating characteristic (ROC) curves were constructed to examine the performance of the ORPI in predicting retrieval of $\geq 4$ oocytes, $\geq 4$ MII oocytes and $\geq 15$ oocytes. The discriminative performance of the model was assessed by the area under the curve (AUC) of the ROC curve.

\section{Results}

The general characteristics of the study population are summarised in Table 1.Of all 734 women, mean age was $30.9 \pm 4.1$ years, mean BMI 24.06 \pm 2.8 , mean AMH level $2.6 \pm 2.0 \mathrm{ng} / \mathrm{mL}$ and mean AFC was $11.5 \pm 5.6$. Mean ORPI calculated was $1.2 \pm 1.3$. The Pearson correlation analysis demonstrated significant $(\mathrm{P}<0.05)$ positive correlations between the ORPI and the total number of oocytes collected and total number of MII oocytes collected. Additionally, other variables of ovarian response i.e. age, $\mathrm{AMH}$ and $\mathrm{AFC}$ showed statistically significant correlation with the variables analysed. However, age and BMI are negatively correlated as depicted in table 2. The performance of the ORPI as a prognostic test was observed using ROC curves. Regarding the probability of collecting $\geq 4$ oocytes, the ROC curve showed an area under the curve of 0.68 (95\% CI: $0.65-0.71)$, indicating that the ORPI had a poor prognostic potency for this point. Setting the threshold of 0.44 , it offered a specificity (51.4\%) and sensitivity $(78.4 \%)$ as illustrated in Figure 1.

In regards to the probability of collecting $\geq 15$ oocytes, ROC curve had an area under the curve of 0.72 (95\% CI: $0.68-0.75)$, indicating that the ORPI had a fair prognostic 
potency. Setting the threshold at 1.28 led to specificity (73.3\%) and sensitivity (66.6\%) as shown in Figure 2. Similarly, figure 3 demonstrates ROC curve for the probability of collecting $\geq 4$ mature oocytes which gave an area under the curve of 0.67 (95\% CI: $0.64-0.70)$, indicating that the ORPI values in this situation had a poor prognostic potency. Setting the threshold at 0.77 depicted specificity of $69 \%$ and sensitivity of $59 \%$. When the ROC curves for all other factors (Age, AMH and AFC) are analysed for their predicting ability for retrieval of $\geq 4$ oocytes,$\geq 15$ oocytes and $\geq 4$ mature oocytes the AUC presented by the ORPI was always higher than age and $\mathrm{AMH}$ and similar to the AUC presented by AFC as depicted in figure 4 .

Table 1: baseline and stimulation characteristics of study population

\begin{tabular}{lll}
\hline & Mean & Std. Deviation \\
\hline No. of Oocytes & 6.48 & 3.504 \\
Matured Oocytes & 4.94 & 2.714 \\
Age & 30.94 & 4.117 \\
BMI & 24.060 & 2.8522 \\
AMH & 2.6058 & 2.01711 \\
AFC & 11.551 & 5.6009 \\
ORPI & 1.1633 & 1.34358 \\
\hline
\end{tabular}

\section{Discussion}

ORPI serves as a perfect tool for having a precise estimate of patient's ovarian response after controlled ovarian stimulation in autologous IVF cycles and optimising treatment. An estimate based only on age is not always sufficient to accurately predict the ovarian response to gonadotropin stimulation, considering that the ovarian response is highly variable even among women of same age group (12). This inter-individual variation is influenced by genetic and environmental factors that primarily determine the size of the pool of primordial follicles at birth and the rate of the pool's decline throughout the reproductive life (13). An ultrasound evaluation of the antral follicle count has gained acceptance as a good predictor of the ovarian response with low intra- and inter-observer variations (14). Based on these observations, a joint analysis of age and the AFC might combine their advantages and compensate for their disadvantages, thus improving the assessment of ovarian function (15).

The combination of different variables of ORPI have resulted in a more precise index to predict ovarian response.
Indeed, the results showed significant correlations $(\mathrm{P}<0.001)$ between the ORPI values and number of oocytes and number of MII oocytes. Our study has shown that ORPI and AFC both have similar predictive value for prediction of ovarian response. Oehninger et al in 2015 concluded similar findings. Contrary to these findings, Nelson et al. (2015) found a better predictive value of AMH versus AFC for oocyte yield. It should be noted that in the Nelson study, 19 assisted reproductive technology centres participated. Because AFC has been shown to have important inter-observer variations, this discrepancy could be explained by the fact that our study was performed in a single centre with only a few operators (16). Despite this test not being universally available and recent alterations in the methods, determination of $\mathrm{AMH}$ can be performed irrespective of day of menstrual cycle with no consistent fluctuation patterns (17).

Cour Freiesleben et al. found that the best prognostic model to predict a low response included $\mathrm{AFC}$ and age. It can be further improved by including serum AMH levels into the calculation of the ORPI (18). In four previous studies, AMH was reported as a stronger predictor than $\operatorname{AFC}(23,24,25,26$, $27,28)$. However, our result was in agreement with four studies that found AFC was superior to AMH for discrimination of ovarian response $(29,30,31,32)$

This prospective study demonstrated that $\mathrm{AMH}, \mathrm{AFC}$ and ORPI were good predictors for high ovarian response and ORPI and AFC were similar $(33,34)$. The addition of age and AMH did not improve the accuracy of AFC. ROC analysis also revealed that AUC for AMH was lower than $\mathrm{AFC}$ and ORPI, but better than basal FSH and age. In contrast to Oliveira et al. study, we found that the new index (ORPI) had no superiority to AFC for prediction of ovarian response (35). On the basis of our knowledge and considering the limited studies in this regard, more well-designed studies are needed for suggesting the potential role of ORPI in the clinical practice for counselling and choosing individual stimulation protocols.

\section{Conclusion}

As no single ovarian reserve marker has $100 \%$ sensitivity and specificity, a combined index of three variables depicted by ovarian reserve prediction index can improve ovarian reserve prediction (36). ORPI serves an excellent counselling tool and key to knowledge enabling proper management of individualized treatment (37).

Table 2: Illustration of correlation between different variables

\begin{tabular}{|c|c|c|c|c|c|c|c|}
\hline & & Matured Ocytes & Age & BMI & $\mathrm{AMH}$ & $\mathrm{AFC}$ & ORPI \\
\hline No. of Oocytes & $\begin{array}{l}\text { Pearson Correlation } \\
\text { Sig. (2-tailed) } \\
\mathrm{N} \\
\end{array}$ & $\begin{array}{l}0.898 \\
0.000 \\
734 \\
\end{array}$ & $\begin{array}{l}-0.141 \\
0.000 \\
734 \\
\end{array}$ & \begin{tabular}{|l|}
-0.020 \\
0.596 \\
734 \\
\end{tabular} & $\begin{array}{l}0.284 \\
0.000 \\
734 \\
\end{array}$ & $\begin{array}{l}0.399 \\
0.000 \\
734 \\
\end{array}$ & $\begin{array}{l}0.295 \\
0.000 \\
734 \\
\end{array}$ \\
\hline Matured Oocytes & Pearson Correlation & & -0.149 & -0.004 & 0.276 & 0.379 & 0.285 \\
\hline & $\begin{array}{l}\text { Sig. (2-tailed) } \\
\mathrm{N}\end{array}$ & & $\begin{array}{l}0.000 \\
734\end{array}$ & $\begin{array}{l}0.913 \\
734\end{array}$ & $\begin{array}{l}0.000 \\
734\end{array}$ & $\begin{array}{l}0.000 \\
734\end{array}$ & $\begin{array}{l}0.000 \\
734\end{array}$ \\
\hline
\end{tabular}




\section{Limitations}

We have not taken pregnancy outcome into consideration.

\section{Acknowledgment}

We thank the consulatants of reproductive medicine

\section{Financial Support}

No financial support was required for the completion of this study.

\section{Conflict of Interest} topic.

The authors declare no conflict of interest in the study

\section{References}

1. Martinez-Conejero JA, Simon C, Pellicer A, Horcajadas JA: Is ovarian stimulation detrimental to the endometrium? Reprod Biomed Online 2007, 15:45-50.

2. Fauser BC, Diedrich K, Devroey P: Predictors of ovarian response: progress towards individualized treatment in ovulation induction and ovarian stimulation. Hum Reprod Update 2008, 14:1-14.

3. Broekmans FJ, Kwee J, Hendriks DJ, Mol BW, Lambalk CB: A systematic review of tests predicting ovarian reserve and IVF outcome. Hum Reprod Update 2006, 12:685-718.

4. Alviggi C, Humaidan P, Ezcurra D: Hormonal, functional and genetic biomarkers in controlled ovarian stimulation: tools for matching patients and protocols. Reprod Biol Endocrinol 2012, $10: 9$.

5. Chang MY, Chiang CH, Chiu TH, Hsieh TT, Soong YK: The antral follicle count predicts the outcome of pregnancy in a controlled ovarian hyperstimulation/intrauterine insemination program. J Assist Reprod Genet 1998, 15:12-17.

6. Lekamge DN, Barry M, Kolo M, Lane M, Gilchrist RB, Tremellen KP: Anti-Mullerian hormone as a predictor of IVF outcome. Reprod Biomed Online 2007, 14:602-610.

7. Jayaprakasan K, Campbell B, Hopkisson J, Johnson I, RaineFenning N: A prospective, comparative analysis of anti-Mullerian hormone, inhibin-B, and three-dimensional ultrasound determinants of ovarian reserve in the prediction of poor response to controlled ovarian stimulation. Fertil Steril 2010, 93:855-864.

8.Oliveira JB, Baruffi RL, Petersen CG, Mauri AL, Nascimento AM, Vagnini L, et al. 2012. A new ovarian response prediction index (ORPI): implications for individualised controlled ovarian stimulation. Reproductive biology and endocrinology: RB\&E 10:94

9.Yovich J, Stanger J, Hinchliffe P: Targeted gonadotrophin stimulation using the PIVET algorithm markedly reduces the risk of OHSS. Reprod Biomed Online 2012, 24:281-292.

10. Nelson SM, Anderson RA, Broekmans FJ, Raine-Fenning N, Fleming R, La Marca A: Anti-Mullerian hormone: clairvoyance or crystal clear? Hum Reprod 2012, 27:631-636.

11. Broer SL, Mol BW, Hendriks D, Broekmans FJ: The role of antimullerian hormone in prediction of outcome after IVF: comparison with the antral follicle count. Fertil Steril 2009, 91:705-714

12. Muttukrishna S, McGarrigle H, Wakim R, Khadum I, Ranieri DM, Serhal P: Antral follicle count, anti-mullerian hormone and inhibin B: predictors of ovarian response in assisted reproductive technology? BJOG 2005, 112:1384-1390.

13. Maheshwari A, Fowler P, Bhattacharya S: Assessment of ovarian reserve- should we perform tests of ovarian reserve routinely? Hum Reprod 2006,21:2729-2735.

14. Younis JS, Jadaon J, Izhaki I, Haddad S, Radin O, Bar-Ami S, Ben-Ami M: A simple multivariate score could predict ovarian reserve, as well as pregnancy rate, in infertile women. Fertil Steril 2010, 94:655-661.

15. Ebner T, Sommergruber M, Moser M, Shebl O, Schreier-Lechner E, Tews G: Basal level of anti-Mullerian hormone is associated with oocyte quality in stimulated cycles. Hum Reprod 2006 21:2022-2026.

16. Riggs RM, Duran EH, Baker MW, Kimble TD, Hobeika E, Yin L, Matos-Bodden L, Leader B, Stadtmauer L: Assessment of ovarian reserve with anti- Mullerian hormone: a comparison of the predictive value of anti- Mullerian hormone, folliclestimulating hormone, inhibin B, and age. Am J Obstet Gynecol 2008, 199:202 e201-208.

17. Aflatoonian A, Oskouian H, Ahmadi S, Oskouian L: Prediction of high ovarian response to controlled ovarian hyperstimulation: anti-Mullerian hormone versus small antral follicle count (2-6 mm). J Assist Reprod Genet 2009, 26:319-325.

18. La Marca A, Stabile G, Artenisio AC, Volpe A: Serum antiMullerian hormone throughout the human menstrual cycle. Hum Reprod 2006, 21:3103-3107.

19.CemFicicioglu, Tayfun Kutlu, Elif Baglam:Early follicular antimullerian harmone as an indicator of ovarian reserve.Fertlity and sterility 2006.85(3):592-6.

20.M McIlveen, J.D.Skull, W.L.Ledger.Evaluation of the utility of multiple endocrine and ultrasound measures of ovarian reservein the prediction of cycle cancellation in a high risk IVF population.Human reproduction 2007.22:778-785.

21.Kunt C,Ozaksit G, Keskin R et al.Anti-Mullerian hormone is a better marker than inhibin $\mathrm{B}$, follicle stimulating hormone,estradiol or antral follicle count in predicting the outcome of in vitro fertilization.Arch Gynecol Obstet.2011;283(6):1415-1421.

22.Joan Carles Arce, Antonio La Marca, Bjarke Mirner Klein.Antimullerian hormone in gonadotropin releasing hormone antagonist cycles:prediction of ovarian response and cumulative treatment outcome in good prognosis patients.Fertil Steril 2013;99:1644-53

23.Van Rooji IA, Broekmans FJ, Te Velde ER et al.Serum antimullerian hormone levels:a novel measure of ovarian reserve.Hum Reprod.2002;17(12):3065-3071.

24.S.Muttukrishna, $\mathrm{H}$ McGarrigle, $\mathrm{R}$ Wakim et al.Antral follicle count, anti-mullerian hormone and inhibin B:predictors of ovarian response in assisted reproductive technology .BJOG volume 112, issue 10 .

25.Lekamge DN, Barry M, kolo M, Gilchrist RB.Anti-mullerian hormone as a predictor of IVF outcome.Reprod Biomed Online.2007;14(5):602-610.

26.Eman A Elgindy, Dahlia O El-Haieg, Azza El-Sebaey.Antimullerian hormone:correlation of early follicular,ovulatory and midluteal levels with ovarian response and cycle outcome in intracytoplasmic sperm injection patients.2008;89(6):1670-1676.

27.Jayaprakasan K, Campbell B, Hopkisson J, Johnson I.A prospective, comparative analysis of anti-mullerian hormone, inhibin B and three dimensional ultrasound determinants of ovarian reserve in the prediction of poor response to controlled ovarian stimulation.Fert Steril.2010;93(3):855-864.

28.Broer SL, Dolleman M, Opmeer BC, Fauser BC.AMH and AFC as predictors of excessive response in controlled ovarian hyperstimulation:a meta-analysis.Hum Reprod Update.2011;17(1):46-54

29.Talia Eldar-Geva, Avraham Ben-Chetrit, Irving M.Spitz, Ron Rabinowitz.Dynamic asays of inhibin B, anti-mullerian hormone and estradiol following FSH stimulation and ovarian ultrasonography as predictors of IVF outcome.2005;20(11):31783183

30.Kwee J, SchatsR, McDonell J,Themmen A.Evaluation of antimullerian hormone as a test for the prediction of ovarian reserve.Fertil Steril.2008;90(3):737-743.

31.Y Himabindu, M Sriharibabu, KK Gopinathan, Usha Satish.Antimullerian hormone and antral follicle count as predictors of ovarian response in assisted reproduction.Journal of Human Reproductive sciences.2013;6(1):27-31.

32.Mutlu MF, Erdem M, Erdem A, et al.Antral follicle count determines poor ovarian response better than anti-mullerian hormone but age is the only predictor for live birth in invitro fertilization cycles.J Assist Reprod Genet.2013;30(5):657-665.

33. Nelson SM, Yates RW, Lyall H, Jamieson M, Traynor I, Gaudoin M, Mitchell P, Ambrose P, Fleming R: Anti-Mullerian hormone- 
based approach to controlled ovarian stimulation for assisted conception. Hum Reprod 2009, 24:867-875.

34.Maryam Eftekhar, Soheila Pourmasumi, Mohammad-Hossein Razi ,Efficacy of rescue ICSI after total fertilization failure in conventional IVF:Journal of Infertility and Reproductive Biology, 2013, Volume 1, Issue 3, Pages: 58-62 58

35. Yates AP, Rustamov O, Roberts SA, Lim HY, Pemberton PW, Smith A, Nardo LG: Anti-Mullerian hormone-tailored stimulation protocols improve outcomes whilst reducing adverse effects and costs of IVF. Hum Reprod 2011, 26:2353-2362.

36.Harpreet Kaur; Prevention of Ovarian Hyperstimulation Syndrome,Journal of Infertility and Reproductive Biology, 2013, Volume 1, Issue 4, Pages: 63-68 63

37. Biasoni V, Patriarca A, Dalmasso P, Bertagna A, Manieri C, Benedetto C, Revelli A: Ovarian sensitivity index is strongly related to circulating $\mathrm{AMH}$ and may be used to predict ovarian response to exogenous gonadotropins in IVF. Reprod Biol Endocrinol 2011, 9:112. 